\title{
Clozapine Associated with Autoimmune Reaction, Fever and Low Level Cardiotoxicity - A Case Report
}

\author{
CHARILAOS GERASIMOU ${ }^{1}$, GEORGIA PHAEDRA VITALI ${ }^{1}$, GEORGE D. VAVOUGIOS ${ }^{2,3}$, \\ CHARALABOS PAPAGEORGIOU ${ }^{4}$, ATHANASIOS DOUZENIS ${ }^{1}$, \\ STYLIANI I. KOKORIS ${ }^{4}$, IOANNIS LIAPPAS ${ }^{5}$ and EMMANOUIL RIZOS ${ }^{1}$ \\ ${ }^{1}$ Second Department of Psychiatry, Attikon General Hospital, \\ National \& Kapodistrian University of Athens, Medical School, Athens, Greece; \\ ${ }^{2}$ Department of Respiratory Medicine, University of Thessaly, School of Medicine, Larissa, Greece; \\ ${ }^{3}$ Athens Naval Hospital, Neurology Clinic, Athens, Greece; \\ ${ }^{4}$ Laboratory of Hematology and Blood Transfusion Department, Attikon General Hospital, \\ National \& Kapodistrian University of Athens, Medical School, Athens, Greece; \\ ${ }^{5}$ First Department of Psychiatry, Eginition Psychiatric Hospital, \\ National \& Kapodistrian University of Athens, Medical School, Athens, Greece
}

\begin{abstract}
Background: Clozapine is a second-generation antipsychotic drug used in treatment-resistant schizophrenia. Fever induced by clozapine is a rather frequent side-effect which usually occurs in the first 4 weeks of treatment. Despite its effectiveness, there are potentially life-threatening adverse effects, such as cardiotoxicity. Case Report: We present the case of a 31year-old caucasian male with refractory schizophrenia who developed benign fever, increase of $C$-reactive protein and high troponin levels, without presenting any other signs to myocarditis, on the 13th day under clozapine treatment, which declined progressively upon discontinuation of the drug. Discussion: This case hints at the presence of initially subclinical cardiotoxicity as an underlying factor in patients developing fever. Conclusion: Taking advantage of more sensitive methods for measuring troponin, clinicians would be promptly aware of this possible side-effect. This would allow for significant reduction of the risk of cardiac dysfunction, further attained by carefully monitoring the patient.
\end{abstract}

This article is freely accessible online.

Correspondence to: E. Rizos, MD, Ph.D., Second Department of Psychiatry, Attikon General Hospital, Rimini 1, Chaidari 124 62, Athens, Greece. Tel: +30 2105832445, Fax: +30 2105326453, e-mail: erizos@med.uoa.gr

Key Words: Cardiotoxicity, fever, clozapine, schizophrenia, case report.
Clozapine is a second-generation antipsychotic drug used in treatment-resistant schizophrenia $(1,2)$. Despite its effectiveness, this drug is not without adverse effects, highgrade fever and cardiotoxicity being among them, through its immunomodulatory effects (3). Clozapine-induced fever has been reported by several studies and a number of plausible explanations for the induction of fever exist including the implication of the immune-modulating effect by increasing soluble tumor necrosis factor (TNF) receptor p55, p75 and soluble interleukin-2 (IL2) receptor $(4,5)$. IL6 has also been suggested to induce fever by elevating the set point of body core temperature via temperature-sensitive neurons in the preoptic area of the hypothalamus (6), for example as a result of pyrogenic cytokines (7). Likewise, drug-induced cardiotoxicity has many underlying mechanisms, affecting the heart directly and, in other cases, indirectly, including the enhancement of immunological reactions, cell apoptosis, formation of free radicals and alterations of hemodynamic flow. Several preclinical and clinical reports have shown the relation of a high incidence of clozapine-induced myocarditis $(3,4,8)$ with immunological-mediated mechanisms.

Here we present the case of a 31-year-old Caucasian male with refractory schizophrenia who developed benign fever, increase of C-reactive protein (CRP) and high sensitivity (Hs)troponin levels, without presenting any other signs of myocarditis, on the 13th day under clozapine treatment, all of which declined progressively upon discontinuation of the drug.

\section{Case Report}

A 31-year-old Caucasian male with no significant past medical history, besides heavy smoking and abusing marijuana, was 
diagnosed with schizophrenia with onset at the age of 22 years, when he experienced auditory hallucinations and delusional ideas of persecution and reference. Over the previous years, he had presented chronic and resistant psychotic symptoms despite treatment with several atypical antipsychotics at therapeutic dosages for adequate durations. Due to the recrudescence of psychotic symptoms, both positive and negative (hallucinations and apathy), the patient was admitted to hospital and clozapine medication was initiated.

Clozapine (25 mg) was introduced at day 1 and titrated to $100 \mathrm{mg}$ within a period of 10 days. The dosage was increased gradually by adding $25 \mathrm{mg}$ every 3 days. After every dose step up, white blood cell count was performed. On the 11th day of treatment with clozapine, a significant elevation of leukocytes $(13,660$ cells/ $\mu \mathrm{l})$ and neutrophils $(10,170$ cells $/ \mu \mathrm{l})$ was observed, indicating potential immune stimulation. On the 13th day, the patient developed fever, rigor and sweating; his maximum temperature was $39.6^{\circ} \mathrm{C}$, and during the day he received three tablets of $500 \mathrm{mg}$ acetaminophen and was rehydrated with 11 of normal saline. Mental state examination showed no alterations. There was no evidence of sensory-perceptual alterations or suicidal ideation. Agitation was treated with $7.5 \mathrm{mg}$ /day lorazepam per os and treatment with clozapine was immediately discontinued. At physical examination, slight tachycardia (140 bpm) and blood pressure of 130/85 $\mathrm{mmHg}$ was found, without any other pathological signs.

In addition, a number of tests were performed. Electrocardiogram revealed sinus tachycardia, normal repolarization and a normal QT interval (QTc) of $340 \mathrm{~ms}$, without alterations of ST or T. Chest X-rays and urinalysis yielded normal results and urine culture was negative. Moreover, blood tests were also within the normal range, except for the white blood count with leukocytosis $(13,100$ cells $/ \mu \mathrm{l})$ and parallel increase of neutrophils $(11,160$ cells $/ \mu \mathrm{l})$. At this time Hs-troponin was also normal, $11.7 \mathrm{pg} / \mathrm{ml}$ (grey zone 14-53 pg/ml, pathological >53 pg/ml).

On the following day (day 14), the patient was feeling better. He was afebrile and blood test revealed a decrease of leucocytes to within the normal range. Surprisingly, we noted an elevation of HS-troponin level to $28.7 \mathrm{pg} / \mathrm{ml}$, raising questions about potential cardiotoxicity. We also found creatine kinase-MB level of $1.25 \mathrm{ng} / \mathrm{ml}$ (normal range $<4.94 \mathrm{ng} / \mathrm{ml})$, procalcitonin level of $0.27 \mathrm{ng} / \mathrm{ml}(<0.5 \mathrm{ng} / \mathrm{ml}$ low possibility of septicemia) and CRP level of $88.60 \mathrm{mg} / \mathrm{l}$ (normal range $0.00-6.00 \mathrm{mg} / \mathrm{l}$ ). More blood tests were performed on days 15 and 17 , showing a gradual decrease of the leucocyte level to within the normal range, and a decrease of CRP to $76.5 \mathrm{mg} / 1$ and $28.4 \mathrm{mg} / \mathrm{l}$, respectively. The HS-troponin level was $20 \mathrm{pg} / \mathrm{ml}$.

At follow-up, on day 32, the patient was in a good medical condition and his blood tests were within normal range.

\section{Discussion}

Clozapine is an antipsychotic agent having immunomodulatory effects. Clozapine-induced-fever is a common side-effect, with an incidence of $0.5 \%$ to $55 \%(9,10)$ and there seems to be a close correlation between fever and the first step of clozapine medication. Recent reports suggest that clozapine stimulates the production of pro-inflammatory cytokines including TNF $\alpha$, IL1 and IL6 $(3,4,11)$. These have also been implicated in causing myocardial depression by direct actions on the myocytes (12).

By considering the above features of our case, we came to believe that there was an ongoing inflammatory process under clozapine titration. We first had to exclude alternate conditions that could explain some of the symptoms. The absence of other typical infectious symptoms and the temporality of leukocytosis, fever and the normalization of troponin upon discontinuing clozapine administration led us to suspect a clozapine-related reaction rather than an infection. Likewise, the absence of cardinal features associated with neuroleptic malignant syndrome (NMS), such as muscle rigidity (typically 'lead pipe'), tremor, abnormal gait, dysphagia, incontinence change of level of consciousness, and confusion, and no alterations of liver enzymes and creatine phosphokinase within normal range made this diagnosis less possible. Moreover, the pathophysiological mechanism by which antipsychotics cause NMS is the blockade of dopaminergic transmission (D2 receptors) in the nigrostriatal system, but in our case NMS is a relatively infrequent side-effect, as clozapine has low affinity for D2 receptors (13). Furthermore, by using the Naranjo causality scale, cardiotoxicity and fever qualified as probable adverse drug reactions to clozapine (14). A discrete elevation of cardiac enzyme (Hs-troponin) indicated the presence of myocardial injury. Negative results from the septic screen, coinciding with a troponin elevation, led us to believe that it could most likely be an onset of myocarditis and the only intervention needed was an immediate withdrawal of clozapine.

A high incidence of fever induced by clozapine represents a therapeutic challenge for clinical doctors, particularly during the initial period of clozapine trial. A standard evaluation including a complete physical examination, complete blood count, assays for creatine kinase and troponins, chest radiography, blood culture and urinalysis should always be considered upon initiation and throughout the treatment.

This case report toughens the need to keep in mind the potential of life-threatening clozapine-induced conditions. Our data are also consistent with previous studies showing the development of fever on day 5 and 20 (median=day 11) upon the initiation of clozapine treatment (9). A CRP increase and a delayed Hs-troponin level elevation should 
raise suspicion of clozapine-related myocardial injury. This finding was shown in a large study by Ronaldson et al., which also showed combining these two parameters improves the sensitivity for detecting symptomatic clozapine-induced cardiotoxicity (15).

This case hints at the presence of initially subclinical cardiotoxicity as an underlying factor in patients developing fever, a rather frequent side-effect, following clozapine initiation. The increase of troponin observed one day after the recession of fever, probably as a late reaction of cardiotoxicity, is remarkable, so the fact that the patient is afebrile should not reassure clinicians. Taking advantage of more sensitive methods for measuring troponin, clinicians would be promptly made aware of this possible side-effect. This would allow a significant reduction of the risk of cardiac dysfunction, further attained by carefully monitoring the patients. More case-control studies are needed in order to clarify the putative model arising from our clinical observations; specifically, a larger sample of patients who develop clozapine-induced fever versus patients commencing clozapine without developing fever as control.

\section{Conflicts of Interest}

The Authors declare no conflicts of interest.

\section{References}

1 Patel SS and Allin MP: Physical complications in early clozapine treatment: a case report and implications for safe monitoring. Ther Adv Psychopharmacol v1(1): 25-29, 2011.

2 Lally J, Gaughram F, Timms P and Curran SR: Treatment-resistant schizophrenia: current insights on the pharmacogenomics of antipsychotics. Pharmgenomics Pers Med 9: 117-129, 2016.

3 Røge R, Møller BK, Andersen CR, Correll CU and Nielsen J: Immunomodulatory effects of clozapine and their clinical implications: what have we learned so far?. Schizophr Res 140(1-3): 204-213, 2012.
4 Abdel-Wahab BA and Metwally ME. Clozapine-induced cardiotoxicity: Role of oxidative stress, tumour necrosis factor alpha and NF-к $\beta$. Cardiovasc Toxicol 15(4): 355-365, 2015.

5 Pollmächer T, Haack M, Schuld A, Kraus T and Hinze-Selch D: Effects of antipsychotic drugs on cytokine networks. J Psychiatr Res 34(6): 369-382, 2000.

6 Conti B, Tabarean I, Andrei C and Bartfai T: Cytokines and fever. Front Biosci 9: 1433-1449, 2004.

7 Pollmächer T, Hinze-Selch D and Mullington J: Effects of clozapine on plasma cytokine and soluble cytokine receptor levels. J Clin Psychopharmacol 16(5): 403-409, 1996.

8 Kilian JG, Kerr K, Lawrence C and Celermajer DS: Myocarditis and cardiomyopathy associated with clozapine. Lancet 354 : 1841-1845, 1999.

9 Jeong SH, Ahn YM, Koo YJ, Kang UG and Kim YS: The characteristics of clozapine-induced fever. Schizophr Res 56: 191-193, 2000

10 Tham JC and Dickson RA: Clozapine-induced fevers and 1-year clozapine discontinuation rate. J Clin Psychiatry 63(10): 880884, 2002.

11 Kluge M, Schuld A, Schacht A, Himmerich H, Dalal MA, Wehmeier PM, Hinze-Selch D, Kraus T, Dittmann RW and Pollmächer T: Effects of clozapine and olanzapine on cytokine systems are closely linked to weight gain and drug-induced fever. Phychoneuroendocr 34(1): 118-128, 2009.

12 Kelly RA and Smith TW: Cytokines and cardiac contractile function. Circulation 95(4): 778-781, 1997.

13 Szota A, Oglodek E and Araszkiewicz A: Fever development in neuroleptic malignant syndrome during treatment with olanzapine and clozapine. Pharmacol Rep 65(2): 279-287, 2013.

14 Naranjo CA, Busto U, Sellers EM, Sandor P, Ruiz I, Roberts EA, Janecek E, Domecq C and Greenblatt DJ: A method for estimating the probability of adverse drug reactions. Clin Pharmacolol Ther 30(2): 239-245, 1981.

15 Ronaldson KJ, Fitzgerald PB, Taylor AJ, Topliss DJ and McNeil JJ: A new monitoring protocol for clozapine-induced myocarditis based on an analysis of 75 cases and 94 controls. Aust NZ J Psychiatry 45(6): 458-465, 2011.

Received September 7, 2016

Revised December 16, 2016

Accepted December 21, 2016 